\title{
Proposta de aprimoramento das técnicas de diagnóstico oftalmológico para motoristas profissionais no Brasil
}

\section{Proposal for improvement of ophthalmological diagnostic techniques for professional drivers in Brazil}

\author{
Eduardo Costa Sá1, Gustavo Ferreira Lessa ${ }^{2}$, Luiz Lippi Rachkorsky²
}

\begin{abstract}
Sá EC, Lessa GF, Rachkorsky LL. Proposta de aprimoramento das técnicas de diagnóstico oftalmológico para motoristas profissionais no Brasil.Saúde, Ética \& Justiça. 2013;18(1):103-9.
\end{abstract}

\begin{abstract}
RESUMO: Introdução: Entre os acidentes automobilísticos que ocorrem em vias públicas, uma parte também pode ser considerada acidentes de trabalho, haja vista que a maioria dos envolvidos se encontra a serviço das empresas. O principal sistema orgânico utilizado no ato de dirigir é o sentido da visão, devendo este ser bem avaliado na obtenção e renovação da habilitação de motoristas profissionais. As estatísticas brasileiras apontam para um aumento global do número de óbitos por acidentes de trânsito, especialmente entre motoristas profissionais de veículos de grande porte entre 1998 e 2008 . Em comparação com os Estados Unidos, o Brasil apresenta um maior índice global de óbitos no trânsito. Pela importância social e econômica desse grupo de motoristas, faz-se necessária a realização de uma avaliação oftalmológica mais estrita que na população em geral. Objetivos: Discutir sobre as diferenças entre os índices mínimos exigidos no exame oftalmológico para obtenção e renovação da carteira de habilitação profissional no Brasil e propor aprimoramento das técnicas de diagnóstico oftalmológico em motoristas profissionais no Brasil. Materiais e Métodos: Foi realizada uma revisão bibliográfica nas bases de dados Pubmed e SciELO em que foram selecionados os artigos publicados em português e inglês entre 1980 e 2011. As palavras-chave utilizadas foram: Técnicas de diagnóstico oftalmológico (Ophthalmological diagnostic techniques), Oftalmopatias (Eye diseases), Exame para habilitação de motoristas (Automobile driver examination) e Condução de veículo (Automobile driving). Foram pesquisados os sites do Código Nacional de Trânsito (Brasil) e do Federal Motor Carrier Safety Administration (Estados Unidos). Como critério de inclusão, foram selecionados apenas artigos sobre motoristas profissionais (categorias C, D e E no Brasil; e grupos A, B e C de motoristas de veículos comerciais nos Estados Unidos). Foram excluídas as publicações relacionadas a motoristas não profissionais (ACC, A e B no Brasil; e veículos não comerciais nos Estados Unidos). Nos países selecionados, foram avaliados dois indicadores utilizados nas técnicas de diagnóstico oftalmológico que fazem parte do exame para obtenção e renovação de habilitação de motoristas profissionais: acuidade visual (AV) e campo visual (CV). O teste de visão cromática é o mesmo nos dois países, não sendo, portanto, objeto de discussão neste estudo. Resultados: No Brasil, os parâmetros utilizados para a mensuração da acuidade visual são utilizados de forma mais rigorosa, assim como o campo visual. Os indicadores exigidos no Brasil devem ser mantidos, especialmente o campo visual, considerando-se que quanto maior a amplitude do campo visual, mais segura é a direção veicular. Sugere-se uma avaliação de triagem das habilidades visuais, cognitivas e motoras na ocasião do exame médico, como pode ser feito com a bateria de testes ADReS (Assessing Driving Related Skills). Softwares como o Driving Health Inventory e a medida do campo visual útil (Useful Field of View - UFOV) mantêm a expectativa de que em alguns anos eles possam ser aplicados nos motoristas profissionais, resultando em maior segurança no trânsito não apenas a esta classe de profissionais, mas a toda população. Conclusão: Os indicadores acuidade visual (AV) e campo visual (CV) utilizados no Brasil são mais estritos em relação aos do país norte-americano. Já a exigência quanto à visão cromática é a mesma nos dois países. Apesar da AV ser um indicador controverso na literatura quanto à capacidade de condução do motorista e de segurança no trânsito, já
\end{abstract}

1. Médico do Serviço de Saúde Ocupacional do Hospital das Clínicas da Faculdade de Medicina da Universidade de São Paulo.

2. Médico residente do programa de Medicina do Trabalho do Hospital das Clínicas da Faculdade de Medicina da Universidade de São Paulo

Endereço para correspondência: Eduardo Costa Sá. Rua Eça de Queiroz, 114, apartamento 131, Vila Mariana, São Paulo SP, CEP 04011-030. E-mail: lessa0301@hotmail.com. Departamento de Medicina Legal, Ética Médica e Medicina Social e do Trabalho da Faculdade de Medicina da Universidade de São Paulo. Instituto Oscar Freire, Rua Teodoro Sampaio, 115 - Cerqueira César, São Paulo, SP. CEP 05405-000. 
Sá EC et al. Proposta de aprimoramento das técnicas de diagnóstico oftalmológico para motoristas profissionais no Brasil.

existem dados consistentes para que seja dada mais atenção ao exame de campo visual. Como proposto por Berson et al ${ }^{3}$, sugere-se, no Brasil, que na avaliação do campo visual sejam analisados $20^{\circ}$ acima e $20^{\circ}$ abaixo do eixo horizontal em cada olho. Propõe-se, ainda, também para esses índices, a padronização dos testes de limiar de visão noturna e de reação ao ofuscamento com determinação prévia de parâmetros de aptidão e inaptidão a serem aplicados pelo médico examinador.

PALAVRAS-CHAVE: Técnicas de diagnóstico oftalmológico; Oftalmopatias; Exame para habilitação de motoristas; Condução de veículo.

\section{INTRODUÇÃO}

$\mathbf{E}$ ntre os acidentes automobilísticos que ocorrem em vias públicas, uma parte também pode ser considerada acidentes de trabalho, haja vista que a maioria dos envolvidos se encontra a serviço das empresas ${ }^{1}$. Um relatório divulgado pela Secretaria de Vigilância em Saúde do Ministério da Saúde $^{2}$ constata que os acidentes de transporte terrestre (ATT) respondem por $26,5 \%$ dos óbitos por causas externas no Brasil, sendo a primeira causa nas populações de 10 a 14 anos e de 40 a 59 anos, e ocupam a segunda posição de mortes por causas externas nas demais faixas etárias. Homens com vinte anos de idade ou mais, residentes nas regiões Sul e Centro-Oeste, são os indivíduos mais vulneráveis à morte por acidentes de trânsito terrestre, sendo que na faixa de 20 a 39 anos, os homens têm um risco seis vezes maior que as mulheres da mesma idade. Todos esses dados demonstram que os acidentes de transporte terrestre se configuram como um problema de saúde pública no Brasil de fundamental importância.

A atividade exercida pelos condutores de veículos comerciais gera um maior potencial de danos em manobras como aceleração, mudança de faixa, resposta à sinalização, julgamento de diversas situações e outros atos potencialmente perigosos. Somando-se estes fatores à capacidade de manobra reduzida em veículos de grande porte, denota-se que indicadores mais estritos devam ser aplicados aos motoristas profissionais, em comparação com a população em geral, para o desempenho seguro no tráfego ${ }^{3}$.

No Brasil, conforme o Anexo I da Resolução número 168 do CONTRAN ${ }^{4}$ de 14 de dezembro de 2004, as habilitações de motorista correspondem às seguintes categorias de veículos:

ACC (autorização para conduzir ciclomotor): Veículo de duas ou três rodas, provido de um motor de combustão interna, cuja cilindrada não exceda a cinquenta centímetros cúbicos e cuja velocidade não exceda a cinquenta quilômetros por hora;

A: Todos os veículos automotores e elétricos, de duas ou três rodas, com ou sem carro lateral;
B: Veículos automotores e elétricos, de quatro rodas, cujo peso bruto total não exceda a três mil e quinhentos quilogramas e cuja lotação não exceda a oito lugares, excluído o do motorista, contemplando a combinação de unidade acoplada, reboque, semirreboque ou articulada, desde que atenda à lotação e capacidade de peso para a categoria;

C: Todos os veículos automotores e elétricos utilizados em transporte de carga, cujo peso bruto total exceda 3500 quilogramas; tratores, máquinas agrícolas e de movimentação de cargas, motor-casa, combinação de veículos em que a unidade acoplada, reboque, semirreboque ou articulada, que não exceda a $6000 \mathrm{Kg}$ de peso bruto total. O condutor habilitado nesta categoria também tem habilitação para conduzir todos os veículos abrangidos pela categoria $\mathrm{B}$;

D: Veículos automotores e elétricos utilizados nos transporte de passageiros, cuja lotação exceda a oito lugares. $\mathrm{O}$ condutor habilitado nesta categoria também tem habilitação para conduzir todos os veículos abrangidos pelas categorias B e C;

E: A unidade tratora se enquadra nas categorias B, $\mathrm{C}$ ou D, cuja unidade acoplada, reboque, semirreboque, articulada, ou ainda com mais de uma unidade tracionada, tenha 6000 quilogramas ou mais de peso bruto total, enquadrados na categoria trailer. O condutor habilitado nesta categoria também tem habilitação para conduzir todos os veículos abrangidos pelas categorias $\mathrm{B}, \mathrm{C}$ e D.

Nos Estados Unidos, o Federal Motor Carrier Safety Administration (FMCSA) ${ }^{5}$, que é o órgão responsável pela prevenção de acidentes de veículos comerciais, normatiza a classificação dos veículos e a classificação das habilitações de motoristas. O FMCSA pertence ao Departamento de Transportes Americano (Department of Transportation - DOT), órgão máximo responsável pelos transportes naquele país. Os veículos automotores são divididos em duas categorias: comerciais e não-comerciais. É considerado veículo comercial o veículo a motor ou conjunto de veículos automotores utilizados no comércio para transporte de passageiros ou de materiais. No país norte-americano, as habilitações de motoristas 
profissionais correspondem aos seguintes grupos de veículos comerciais:

Grupo A (Combinação de veículos): Qualquer combinação de veículos com um peso bruto total de $11.794 \mathrm{~kg}$ ou mais (26.001 libras ou mais), desde que o peso bruto total do veículo a ser rebocado seja superior a $4.536 \mathrm{~kg}$ (10.000 libras);

Grupo B (Veículo pesado): Qualquer veículo único com um peso bruto total de $11.794 \mathrm{~kg}$ ou mais (26.001 libras ou mais) ou qualquer veículo que reboque um peso bruto total não superior a $4.536 \mathrm{~kg}$ (10.000 libras);

Grupo C (Veículo pequeno): Qualquer veículo isolado, ou combinação de veículos, que não se encaixe na definição do Grupo A ou do Grupo B, mas que, ou é projetado para transportar 16 passageiros ou mais, incluindo o motorista, ou é utilizado no transporte de materiais considerados perigosos segundo a Lei de Transporte de Materiais Perigosos, que exige sinalização do veículo conforme o regulamento de materiais perigosos.

Para o ato de dirigir, apenas uma visão adequada não é suficiente para uma direção segura. Além de perceber com precisão todos os elementos presentes no cenário de tráfego, o motorista deve também interpretálos corretamente e reagir a eles de forma eficiente com uma resposta neuromuscular aplicada aos comandos do automóvel, muitas vezes em espaços de tempo muito curtos e em alta velocidade ${ }^{6}$.

O exame oftalmológico é de alta relevância na avaliação de motoristas profissionais e deve ser realizado de maneira adequada com o objetivo de diminuir os acidentes de trânsito causados por alterações visuais. No Brasil, o médico perito examinador deve basear a avaliação oftalmológica nos seguintes parâmetros: acuidade visual (AV), campo visual (CV), visão cromática e teste de ofuscamento e visão noturna. De acordo com Adura $^{6}$, os portadores de morbidades oftalmológicas guiam veículos com menos segurança e correm maior risco de provocar acidentes.

Os índices mínimos exigidos na avaliação oftalmológica no Brasil, segundo a Resolução 267/08 do CONTRAN ${ }^{7}$, e nos Estados Unidos, segundo o FMCSA ${ }^{5}$, estão apresentados na Tabela 1.

Tabela 1. Índices mínimos exigidos no Brasil e nos Estados Unidos para avaliação oftalmológica de motoristas profissionais

\begin{tabular}{|c|c|c|}
\hline Índices Mínimos Exigidos & Brasil* & Estados Unidos** \\
\hline Acuidade Visual & $\begin{array}{l}\geq 20 / 30 \text { em cada olho ou } \geq 20 / 40 \text { em um } \\
\text { olho e visão binocular } \geq 20 / 25\end{array}$ & $\begin{array}{l}\geq 20 / 40 \text { em cada olho ou visão } \\
\text { binocular } \geq 20 / 40\end{array}$ \\
\hline Campo Visual & $\geq 120^{\circ}$ em cada olho & $\geq 70^{\circ}$ em cada olho \\
\hline Visão Cromática & $\begin{array}{l}\text { Disfunção das cores verde, amarela e } \\
\text { vermelha }\end{array}$ & $\begin{array}{l}\text { Disfunção das cores verde, amarela e } \\
\text { vermelha }\end{array}$ \\
\hline Limiar de Visão Noturna & Possuir visão em baixa luminosidade & Não especificado \\
\hline Reação ao Ofuscamento & Recuperação após ofuscamento direto & Não especificado \\
\hline
\end{tabular}

Waiselfisz ${ }^{8}$, em 2011, divulgou um relatório com estatísticas de acidentes automobilísticos no Brasil de 1998 a 2008. No estudo, o autor demonstra o crescimento do número de óbitos por acidentes de trânsito em cada categoria de veículos (divididas em: ciclistas, motociclistas, automóveis, caminhões, ônibus, outros e não especificado). Com exceção dos pedestres, todas as outras categorias tiveram um aumento do número de óbitos. Neste período de dez anos, particularmente, o índice de óbitos por cem mil habitantes nas categorias caminhão e ônibus aumentou $183 \%$ e $73,8 \%$, respectivamente. Este dado reforça a necessidade de uma avaliação oftalmológica mais rigorosa nessas categorias de condutores.

Em 2008, a Organização Mundial da Saúde $(\mathrm{OMS})^{9}$ realizou uma pesquisa que compilava dados recentes (a maioria, do ano 2007) sobre acidentes de trânsito em 178 países. O relatório da pesquisa, chamado de "Estado Global de Segurança nas Estradas", foi o primeiro estudo sobre acidentes de trânsito no mundo. O estudo dá uma visão panorâmica atual das mortes no trânsito no mundo. Entre outras variáveis, a pesquisa apresenta o índice de mortes no trânsito por cem mil habitantes em cada país. O Brasil, em 2006, apresentou um índice de 18,3 mortes/100.000 habitantes, enquanto a mediana mundial foi de 16,6. Significativamente menor, o índice apresentado pelos Estados Unidos, no mesmo ano, foi de 13,9 mortes/100.000 habitantes. Vários fatores influenciam nesses resultados, como o rigor de cada legislação, padronização dos itens de segurança dos veículos, existência de meios de transporte coletivo e 
Sá EC et al. Proposta de aprimoramento das técnicas de diagnóstico oftalmológico para motoristas profissionais no Brasil.

atendimento pré-hospitalar nas vias públicas.

Com base na análise retrospectiva de acidentes e estatísticas de mortalidade realizada nos Estados Unidos, os acidentes de veículos comerciais representam um maior risco no que tange a danos corporais e materiais ${ }^{3}$. É por esta razão que, mundialmente, deve existir um critério mais estrito, especialmente entre os condutores de veículos comerciais, tanto na obtenção da habilitação de motorista, quanto em sua renovação.

\section{OBJETIVOS}

Identificar as principais técnicas existentes na avaliação oftalmológica de motoristas profissionais e suas diferenças entre Brasil e Estados Unidos;

Propor aprimoramento das técnicas de diagnóstico oftalmológico em motoristas profissionais no Brasil.

\section{MATERIAIS E MÉTODOS}

Foi realizada uma revisão bibliográfica nas bases de dados Pubmed e SciELO em que foram selecionados os artigos publicados em português e inglês entre 1980 e 2011. As palavras-chave utilizadas foram: Técnicas de diagnóstico oftalmológico (Ophthalmological diagnostic techniques), Oftalmopatias (Eye diseases), Exame para habilitação de motoristas (Automobile driver examination) e Condução de veículo (Automobile driving). Foram pesquisados os sites do Código Nacional de Trânsito (Brasil) - www.denatran.gov.br/contran.htm - e do Federal Motor Carrier Safety Administration (Estados Unidos) - www.fmcsa.dot.gov. Como critério de inclusão, foram selecionados apenas artigos sobre motoristas profissionais (categorias C, D e E no Brasil; e grupos A, $\mathrm{B}$ e $\mathrm{C}$ de motoristas de veículos comerciais nos Estados Unidos). Foram excluídas as publicações relacionadas a motoristas não profissionais (ACC, A e B no Brasil; e veículos não comerciais nos Estados Unidos). Nos países selecionados, foram avaliados dois indicadores utilizados nas técnicas de diagnóstico oftalmológico que fazem parte do exame para obtenção e renovação de habilitação de motoristas profissionais: acuidade visual (AV) e campo visual $(\mathrm{CV})$. O teste de visão cromática é o mesmo nos dois países, não sendo objeto de discussão neste estudo.

\section{RESULTADOS}

Observa-se que ambas as legislações fazem referência aos seguintes indicadores: acuidade visual central (AV), visão periférica na isóptera horizontal e visão cromática. A legislação brasileira requer, ainda, visão em baixa luminosidade e recuperação após ofuscamento direto.
Não há nenhuma padronização quanto à taxa de luminosidade e quanto ao tempo de recuperação ao ofuscamento, cuja intensidade e tempo máximo de reação ao estímulo não são especificados. A legislação americana não menciona nenhuma exigência quanto a estes indicadores. Nenhum dos dois países exige testes para avaliar visão estereoscópica, que é responsável pela visão binocular (identificação da profundidade dos objetos no espaço).

A acuidade visual central é exigida de forma mais estrita no Brasil, em comparação com os Estados Unidos. Em ambos os países o método de avaliação é o mesmo, sendo ele a tabela de Snellen. Quando o exame é realizado com uso de lentes corretivas, faz-se uma restrição na habilitação de motorista, em que o mesmo deve usá-las durante a condução do veículo, tanto no Brasil, quanto nos Estados Unidos.

O teste de visão cromática exige, em ambos os países, que o candidato reconheça as cores verde, vermelha e amarela, não sendo importante o diagnóstico de discromatopsias. Adura ${ }^{6}$ afirma que a avaliação pode ser feita por meio de sinais luminosos, lâmpadas coloridas, cartolinas ou novelos de lã.

\section{DISCUSSÃO}

Currie et al. ${ }^{10}$ afirmam que o teste de acuidade visual com a tabela de Snellen é um fraco preditor da capacidade individual de dirigir. A Academia Americana de Oftalmologia ${ }^{11}$ constata que não existem dados consistentes que confirmem que a acuidade visual seja um preditor válido sobre a capacidade de condução e segurança no trânsito. A mesma fonte ainda afirma que existem indicadores de direção segura mais acurados que a acuidade visual, além de que não há base para a exigência de acuidade visual maior ou igual a 20/40, que é atualmente a exigência do FMCSA.

Estudos apontam o campo visual como sendo um indicador mais importante que a acuidade visual central durante o ato de dirigir ${ }^{12,13}$. Neste quesito, a legislação brasileira também é mais estrita, exigindo uma visão na isóptera horizontal de $120^{\circ} \mathrm{em}$ cada olho, frente a $70^{\circ}$ (em cada olho na isóptera horizontal) nos Estados Unidos. Berson et al. ${ }^{3}$ recomendam que se adote no país norte-americano a exigência de campo visual de $120^{\circ} \mathrm{em}$ cada olho na isóptera horizontal, como é feito no Brasil, além de $20^{\circ}$ acima e $20^{\circ}$ abaixo da isóptera horizontal em cada olho. Estes indicadores devem ser avaliados por meio do teste de confrontação de campo visual em cada olho separadamente. Indivíduos que obtivessem resultados fora dos limites aceitáveis, ou fossem diagnosticados como tendo uma doença que pode comprometer o campo visual (como glaucoma, retinite pigmentosa, acidente vascular cerebral ou tumor cerebral) seriam obrigados a 
ter uma avaliação visual completa por um oftalmologista (ou optometrista, profissional, ao qual é permitido fazer o exame oftalmológico de obtenção de carteira de habilitação nos Estados Unidos) a fim se fazer testes mais acurados de campo visual, seguidos por um relatório sobre a aptidão do motorista para dirigir veículos comerciais.

Entretanto, não existe consenso sobre a amplitude de campo visual necessária para uma condução veicular segura. Alguns autores ${ }^{12,13,14}$ defendem que um campo visual reduzido pode ser compensado por outros mecanismos, mantendo a segurança na direção de veículos. O desempenho no trânsito de um motorista com campo visual restrito, porém com capacidade de verificação do ambiente excelente, por exemplo, pode ser mais seguro do que o de um motorista com campo visual completo, mas sem rotação do pescoço que permita a visualização ampla do cenário de tráfego ${ }^{11}$.

Berson et al. $^{3}$ criticam o fato de o exame oftalmológico nos Estados Unidos avaliar apenas a visão estática, e afirmam que deveriam ser usados testes que apontassem variáveis visuais, cognitivas e motoras, como em simuladores de vídeo. Durante a condução, a atenção é requerida de diferentes maneiras. O motorista deve possuir atenção seletiva, que é a capacidade de priorizar os estímulos visuais e focar apenas naquele mais importante, com a finalidade de responder a estímulos urgentes (como sinais de trânsito), sem se distrair com aqueles irrelevantes (como anúncios comerciais à margem da estrada) ${ }^{14}$. Além disso, os motoristas devem possuir atenção dividida, que é a capacidade de se concentrar nas múltiplas tarefas exigidas durante a condução. $\mathrm{O}$ funcionamento da atenção pode diminuir com a idade ${ }^{15}$, sendo que a redução da atenção dividida tem se mostrado mais pronunciada do que a atenção seletiva $^{16}$.

$\mathrm{O}$ instrumento mais amplamente estudado para detecção de comprometimento de atenção dividida e de atenção seletiva tem sido o campo de visão útil (Useful Field of View - UFOV), um teste computadorizado, cujos resultados vêm sendo correlacionados com o risco de acidentes em motoristas mais velhos ${ }^{11}$.

O teste de campo visual útil foi desenvolvido para avaliar a capacidade do indivíduo de processar e reagir em tempo hábil a vários eventos que ocorrem simultaneamente. Ele define o campo visual em situações nas quais obstáculos surgem rapidamente, e o indivíduo deve detectá-los sem movimentar os olhos ou a cabeça. O teste inclui três variáveis: atenção dividida, atenção seletiva e velocidade de processamento. Uma redução no UFOV tem sido associada a um envolvimento maior em acidentes, enquanto que uma redução moderada da acuidade visual, sensibilidade de contraste e no campo visual não foi associada ao mesmo desfecho ${ }^{17}$.

$\mathrm{O}$ teste UFOV depende tanto de habilidades visuais quanto cognitivas, fornecendo uma medida mais abrangente dos status funcional e visual se comparado a testes sensoriais ou cognitivos isoladamente ${ }^{13}$. É um bom preditor do desempenho de condução em pacientes com acuidade visual normal, mas cognição comprometida ${ }^{15}$, além de ser melhor do que a idade cronológica para a identificação de motoristas em risco de acidentes ${ }^{12}$. Em alguns estados americanos o UFOV tem sido usado experimentalmente. Em outros, ele é associado com outros testes de triagem, simuladores ou usado informalmente para programas de educação de motoristas ${ }^{13,15}$. Devido ao limitado número de estudos a respeito do teste, além do alto custo e tempo necessário para o exame, este teste ainda não é utilizado em larga escala nos Estados Unidos ou no Brasil.

Estudos têm sido feitos sobre uma bateria de testes chamada de Avaliação de Habilidades Relacionadas à Condução (Assessing Driving Related Skills - ADReS). Ela demanda materiais simples (tabela de Snellen, papel, lápis, fita métrica e cronômetro), tendo fácil aplicabilidade. Embora ainda sejam necessários mais estudos baseados em evidências para relacionar estes testes com risco de acidentes de trânsito, esta ferramenta pode rastrear o surgimento de déficits das funções visuais, cognitivas ou motoras ${ }^{18}$.

Similar à bateria ADReS, o Driving Health Inventory ${ }^{\circledR}{ }^{16}$, que inclui o teste UFOV, é um software utilizado como rastreamento de déficits funcionais. A avaliação é padronizada e rápida, determinando o grau de eventuais incapacidades funcionais para uma direção segura. As funções avaliadas no teste são: acuidade visual, visão de contraste, força e resistência das pernas, flexibilidade da cabeça e pescoço, planejamento de rotas, memórias de curto prazo e de trabalho, reconhecimento da falta de informações, busca visual com atenção dividida e velocidade de processamento de informação visual $^{16}$

Na Tabela 2 estão apresentadas as propostas de aprimoramento das técnicas de diagnóstico oftalmológico em motoristas profissionais no Brasil quanto aos índices de: campo visual, limiar de visão noturna e reação ao ofuscamento.

Os exames de habilitação em categorias de motoristas profissionais deveriam incluir uma avaliação de funções cognitivas, motoras e visuais. A utilização em larga escala de um software em que sejam avaliadas estas funções ainda é uma promessa, porém deveriam ser utilizadas baterias de testes simples e acessíveis, como a bateria ADReS, para se fazer um rastreamento mais sensível, principalmente de incapacidades motoras e cognitivas. Contudo, o crescente número de estudos sobre o tema mantém a expectativa de que a saúde e a segurança dos motoristas profissionais ganhem mais atenção da sociedade e da comunidade científica. 
Sá EC et al. Proposta de aprimoramento das técnicas de diagnóstico oftalmológico para motoristas profissionais no Brasil.

Tabela 2. Propostas de aprimoramento das técnicas de diagnóstico oftalmológico em motoristas profissionais no Brasil

\begin{tabular}{ll} 
Índices Mínimos & Brasil \\
\hline Acuidade Visual & Manutenção do critério atual \\
\hline Campo Visual & Acrescentar $20^{\circ}$ acima e $20^{\circ}$ abaixo na isóptera horizontal em cada olho \\
\hline Visão Cromática & Manutenção do critério atual \\
\hline Limiar de Visão Noturna & Adoção de parâmetros de luminosidade (intensidade e tempo do estímulo) e índice mínimo exigido \\
\hline Reação ao Ofuscamento & Adoção de parâmetros de luminosidade (intensidade e tempo do estímulo) e índice mínimo exigido
\end{tabular}

\section{CONCLUSÃO}

Os indicadores acuidade visual (AV) e campo visual $(\mathrm{CV})$ utilizados no Brasil são mais estritos em relação aos do país norte-americano. Já a exigência quanto à visão cromática é a mesma nos dois países. Apesar da AV ser um indicador controverso na literatura quanto à capacidade condução do motorista e segurança no trânsito, já existem dados consistentes para que seja dada mais atenção ao exame de campo visual.

Como sugestão a ser aplicada no Brasil, propõese que na avaliação do campo visual sejam analisados $20^{\circ}$ acima e $20^{\circ}$ abaixo do eixo horizontal em cada olho, como proposto por Berson et al. $^{3}$. Propõe-se, ainda, também para esses índices, a padronização dos testes de limiar de visão noturna e de reação ao ofuscamento com determinação prévia de parâmetros de aptidão e inaptidão a serem aplicados pelo médico examinador.

Sá EC, Lessa GF, Rachkorsky LL. Proposal for improvement of ophthalmological diagnostic techniques for professional drivers in Brazil. Saúde, Ética \& Justiça. 2013;18(1):103-9.

\begin{abstract}
Introduction: Part of the motor vehicle accidents that occur on public roads may also be considered occupational accidents, given that the majority of those involved occur while in the service of companies. The main sense system used to drive is that of eyesight, which must be carefully evaluated in obtaining and renewing a of professional drivers license. Brazilian statistics indicate an overall increase in the number of deaths from traffic accidents, especially among professional drivers of large vehicles between 1998 and 2008. Compared to the United States, Brazil has a higher overall rate of deaths in road accidents. By the social and economic importance of this group of drivers, it is necessary to perform a more stringent ophthalmic evaluation than that performed in general population. Objective: Discuss the differences between the minimum indicators required by ophthalmological examination for obtaining and renewing professional driver's license in Brazil and propose an improvement of ophthalmological diagnostic techniques for professional drivers in Brazil. Materials and Methods: We performed a literature review on the PubMed and SciELO databases and selected articles published in Portuguese and English between 1980 and 2011. The keywords used were: Ophthalmological diagnostic techniques, Eye diseases, Automobile driver examination and Automobile driving. As inclusion criteria, only articles were selected about professional drivers (categories C, D and E in Brazil, and groups A, B and $\mathrm{C}$ of commercial vehicle drivers in the United States). We excluded publications related to non-professional drivers (ACC, A and B in Brazil, and non-commercial vehicles in the United States). In both countries two indicators were assessed that had been used in ophthalmological diagnostic techniques as part of the examination for obtaining and renewing driver's licenses of professional drivers: visual acuity (VA) and visual field (VF). The color vision test is the same in both countries, therefore not being a subject of discussion in this study. Results: In Brazil, the parameters for measurement of visual acuity, such as those of the visual field, are used more strictly. The indicators required in Brazil must be maintained, especially the visual field, considering that the higher the amplitude of the visual field, the safer it is for vehicle guidance. We suggest there be a screening evaluation of visual, cognitive and motor skills included in the medical examination, as can be done with the ADReS (Assessing Driving Related Skills) battery tests. With softwares like the Driving Health Inventory and the measurement of the Useful Field of View (UFOV) the expectation can be maintained that in a few years they will be applied with professional drivers, resulting in greater road safety not only to this class of professionals, but to the entire population. Conclusion: Indicators of visual acuity and visual field used in Brazil are stricter regarding those used in the United States of America. The requirement of color vision is the same in both countries. Despite vision acuity being controversial in the literature regarding driving ability and traffic safety, there are consistent data that provide evidence that more attention should be given to visual field. The authors propose, as a suggestion to be applied in Brazil, that the assessment of the visual field should be analyzed 20 degrees above and 20 degrees below the horizontal axis in each eye, as proposed by Berson et $a l^{4}$. It is further proposed, also for these indexes, that there be standardization of the tests of threshold of night vision and reaction to glare with previous determination of parameters of ability and inability to be applied by the medical examiner.
\end{abstract}

KEYWORDS: Diagnostic techniques, ophthalmological; Eye diseases; Automobile driver examination; Automobile driving. 


\section{REFERÊNCIAS}

1. Silva LG. O trabalho dos motoristas de caminhão: a relação entre atividade, vínculo empregatício e acidentes de trabalho [Dissertação]. São Paulo: Universidade de São Paulo, Faculdade de Saúde Pública; 2011. Disponível em: http://www.teses.usp.br/teses/disponiveis/6/6134/ tde-24022011-111659/?\&lang=en

2. Brasil. Ministério da Saúde. Secretaria de Vigilância em Saúde. Epidemiologia das causas externas no Brasil: mortalidade por acidentes e violências no período de 2000 a 2009. Brasília; 2010. p. 225-49 [citado em 10 dez. 2012]. Disponível em: http://portal.saude.gov.br/portal/ arquivos/pdf/cap_11_saude_brasil_2010.pdf.

3. Berson FG, Kuperwaser MC, Aiello LP, Rosenberg JW. Visual requirements for commercial drivers. Washington (DC): Federal Highway Administration, Office of Motor Carriers; 1998.

4. Brasil. Ministério das Cidades. Conselho Nacional de Trânsito (CONTRAN). Resolução n ${ }^{\circ} 168$ de 14 de dezembro de 2004 - Estabelece Normas e Procedimentos para a formação de condutores de veículos automotores e elétricos, a realização dos exames, a expedição de documentos de habilitação, os cursos de formação, de reciclagem e dá outras providências [citado em 10 dez. 2012]. Diário Oficial da União. 2005 mar. 22, Seção 1, p.36-44. Disponível em: http://www. denatran.gov.br/download/Resolucoes/RESOLUCAO_ CONTRAN_347_10.pdf

5. United States. Department of Transportation. Federal Motor Carrier Safety Administration. Physical qualifications for drivers - Part 391. Subpart E, § 391.41 [cited 2012 Dec 10]. Available from: http://www. fmcsa.dot.gov/rules-regulations/administration/fmcsr/ fmcsrruletext.aspx?reg=391.41.

6. Adura FE, Sabbag AF. Manual para o médico perito examinador de candidatos a motorista. 4a ed. São Paulo: Abramet; 2011.

7. Brasil. Ministério das Cidades. Conselho Nacional de Trânsito (CONTRAN). Resolução n ${ }^{\circ} 267$ de 15 de fevereiro de 2008. Dispõe sobre o exame de aptidão física e mental, a avaliação psicológica e o credenciamento das entidades públicas e privadas que tratam o art. 147, I e $\S \S 1^{\circ}$ a $4^{\circ}$ e o art. 148 do Código de Trânsito Brasileiro. Brasília: DENATRAN; 2008 [citado em 10 dez 2012]. Disponível em: http://www.denatran.gov.br/download/ Resolucoes/RESOLUCAO_CONTRAN_267.pdf.

8. Waiselfisz JJ. Mapa da violência 2011. Os jovens do
Brasil. São Paulo: Instituto Sangari; 2011.

9. World Health Organization. Department of Violence \& Injury Prevention \& Disability (VIP). Global Status Report on Road Safety - Time for Action. Switzerland; 2009.

10. Currie Z, Bhan A, Pepper I. Reliability of Snellen charts for testing visual acuity for driving: prospective study and postal questionnaire. BMJ. 2000; 321(7267): 990-2. DOI: http://dx.doi.org/10.1136/bmj.321.7267.990

11. American Academy of Ophthalmology. Policy Statement. Vision Requirements for Driving, 2006 [cited 2012 Dec 10]. Available from: http://www.aao.org/about/policy/ upload/AAODrivingPolicyWebcopy.pdf

12. Owsley C., McGwin J. G. Vision Impairment and Driving. Surv Ophthalmol. 1999; 43(6): 535-50. DOI: http://dx.doi.org/10.1016/S0039-6257(99)00035-1

13. Johnson CA, Keltner JL. Incidence of visual field loss in 20,000 eyes and its relationship to driving performance. Arch Ophthalmol. 1983;101(1):371-5. DOI: http://dx.doi. org/10.1001/archopht.1983.01040010371002

14. Madden DJ, Turkington TG, Provenzale JM, Hawk TC, Hoffman JM, Coleman RE. Selective and divided visual attention: age-related changes in regional cerebral blood flow measured by $\mathrm{H} 215 \mathrm{O}$ PET. Hum Brain Mapp. 1997; 5(6): 389-409. DOI: http://dx.doi. org/10.1002/(SICI) 1097-0193(1997)5:6<389::AIDHBM1 $>3.0 . \mathrm{CO} ; 2-\% 23$

15. Hartley AA. Attention. In: Craik FIM, Salthouse TA, editors. The handbook of aging and cognition. Hillsdale, NJ: Erlbaum; 1992. p.3-50.

16. Driving Health Inventory - including UFOV [programa de computador]. Quakertown, PA: TransAnalytics Health \& Safety Services; 2011 [cited 2012 Dec 10]. Available from: www.drivinghealth.com/screening.htm.

17. Ball K, Owsley C, Sloane ME, Roenker DL, Bruni JR. Visual attention problems as a predictor of vehicle crashes in older drivers. Invest Ophthalmol Vis Sci. 1993;34(11):3110-23. Available from: http://www.iovs. org/content/34/11/3110.full.pdf

18. Physician's Guide to Assessing and Counseling Older Drivers. 2nd ed. Chicago, IL: American Medical Association; 2010 [cited 2012 Dec 10]. Available from: http://www.ama-assn.org/resources/doc/public-health/ older-drivers-guide.pdf 